\title{
Cryotherapy is associated with improved clinical outcomes of sorafenib for the treatment of advanced hepatocellular carcinoma
}

\author{
YONGPING YANG $^{1,2^{*}}$, YINYING LU $^{1 *}$, CHUNPING WANG $^{1}$, WENLIN BAI $^{1}$, JIANHUI QU $^{1,2}$, YAN CHEN $^{1}$, \\ XIUJUAN CHANG ${ }^{1}$, LINJING AN ${ }^{1}$, LIN ZHOU ${ }^{1}$, ZHEN ZENG ${ }^{1}$, MIN LOU ${ }^{1}$ and JIYUN LV ${ }^{1}$ \\ ${ }^{1}$ Center of Therapeutic Research for Hepatocellular Carcinoma, Beijing 302nd Hospital; \\ ${ }^{2}$ Beijing Institute of Infectious Diseases, Beijing 100039, P.R. China
}

Received September 6, 2011; Accepted November 11, 2011

DOI: $10.3892 / \mathrm{etm} .2011 .398$

\begin{abstract}
Sorafenib may prolong survival in patients with advanced hepatocellular carcinoma (HCC), but with limited efficacy. The present study aimed to assess the safety and efficacy of sorafenib combined with cryotherapy (cryoRx) for the treatment of advanced HCC. A total of 104 patients met the following criteria: advanced HCC without distant metastasis, presence of portal vein thrombosis, Child-Pugh class A or B and life expectancy of at least 12 weeks. All patients were randomly assigned to sorafenib and cryoRx $(n=52)$ or sorafenib-alone $(n=52)$ treatment groups. The primary end-point of the study was overall survival (OS). The secondary end-points included time to progression (TTP) and tolerability. Microvessel density (MVD) was assessed following immunostaining for CD34. In a median of 10.5 (4-26) months follow-up, the median OS was 12.5 months (95\% CI 10.6-16.4) in the combination therapy vs. 8.6 months $(7.3-10.4)$ in the sorafenib-alone $(\mathrm{P}=0.01)$ group. The median TTP was 9.5 months (8.4-13.5) in the combination therapy vs. 5.3 months (3.8-6.9) in the sorafenib alone $(\mathrm{P}=0.02)$ group. CryoRx was an independent factor associated with improved clinical outcomes of sorafenib for the treatment of advanced HCC. Patients with low intratumoral MVD receiving the combination therapy exhibited a significantly longer median TTP and OS compared to those receiving sorafenib. High intratumoral MVD was an independent predictor of poor responses to sorafenib for advanced HCC. Compared with previous reports of sorafenib-related adverse drug reactions (ADRs), cryoRx did not further increase the frequency and degree of sorafenib-related ADRs. In conclusion, compared to sorafenib alone, the addition of cryoRx to sorafenib significantly improves the clinical outcomes of sorafenib for the treatment of advanced
\end{abstract}

Correspondence to: Dr Yongping Yang, Center of Therapeutic Research for Hepatocellular Carcinoma, Beijing 302nd Hospital, 100 Xisihuan Middle Road, Beijing 100039, P.R. China

E-mail: yongpingyang@hotmail.com

*Contributed equally

Key words: hepatocellular carcinoma, sorafenib, cryotherapy, microvessel density, efficacy, safety
HCC with acceptable tolerance and similar safety profiles as previously reported. High intratumoral MVD is predictive of poor responses to sorafenib in advanced HCC patients.

\section{Introduction}

Hepatocellular carcinoma (HCC) is the third most common cause of cancer-related mortality worldwide (1) and the second most common cause of cancer-related mortality in China (2). The majority of patients with advanced HCC at the time of initial diagnosis exhibit poor outcomes (3). In China, HCC is most commonly caused by infection with the hepatitis $\mathrm{B}$ virus (HBV) (4). The incidence of HCC has increased in recent years, largely owing to chronic HBV infection-related liver cirrhosis (5). Therapeutic options are stage-dependent $(6,7)$. Only approximately $30 \%$ of patients who present with early stage tumors undergo resection, liver transplantation and percutaneous ablation, due to various factors, such as multifocal tumor and poor liver function resulting from underlying cirrhosis (8-10). Until recently, no effective treatment was available for these conditions (11). Sorafenib is a newly developed, molecular targeted agent. This multikinase inhibitor has demonstrated significant survival benefits in phase III trials for patients with advanced HCC $(12,13)$. However, its efficacy remains moderate and certain patients continue to display a short period of survival following treatment (14). The mechanisms causing certain patients to become refractory to sorafenib are, at present, unclear. High intratumoral microvessel density (MVD) has been associated with a higher level of activity along the vascular endothelial growth factor (VEGF)/VEGF receptor (VEGFR) signaling pathway. As such, the presence of high intratumoral MVD in advanced HCC patients may be associated with a positive response to sorafenib treatment. However, it remains unknown as to whether the presence of high intratumoral MVD is capable of effecting responses to sorafenib treatment in advanced HCC patients. Previous findings have provided a strong rationale for combining the two treatment modalities. In mice with implanted renal tumors, the combination of radiofrequency ablation (RFA) and sorafenib has been found to cause an increase in the efficacy of tumor ablation that is dependent on the dose of sorafenib (15). Although the value of cryotherapy (cryoRx) in HCC is not yet as well established as that of RFA, cryoRx has been found to be more advantageous 
for improving immunity following treatment compared to RFA. Osada et al found that not only the local tumor, but also the adjacent tumor tissue was necrotic and shrunken in HCC patients following cryoablation, which was regarded as ectopic tumor suppression (16). This response may be associated with the release of tumor antigens, resulting in the host production of anti-tumor antibodies (17). The majority of the bias against cryoRx for HCC is based on the theoretical risk associated with a cryoablation modality that does not employ cauterization-like, heat-based ablation therapy and as a result of the large probes, which may cause serious bleeding when removed (18). The experimental evaluation has identified no significant difference among the hemorrhages encountered following an ablation with a single RF probe versus a single cryoprobe (19). Therefore, this technology has been used extensively in open surgical settings and, more recently, applied percutaneously to treat renal tumors and liver metastases $(20,21)$. Nevertheless, the efficacy of the use of cryoRx for the improvement of clinical outcomes of sorafenib for the treatment of advanced HCC is, at present, unknown. The aim of this study was to confirm the efficacy and safety of sorafenib combined with cryoRx for the treatment of advanced HCC, as well as the croyablation tumor burden impact for sorafenib therapy responses.

\section{Patients and methods}

Patients. Based on the Barcelona Clinic Liver Cancer (BCLC) staging classification (7), 296 consecutive patients with HBV-related advanced HCC were screened between July 2008 and July 2010, at the Center of Therapeutic Research for Hepatocellular Carcinoma (Beijing, China). A total of 57 patients were classified as Child-Pugh C, 38 patients were classified as Child-Pugh B8 or B9, with serum bilirubin levels of $>51.3 \mu \mathrm{mol} / 1$. The life expectancy of 23 patients was $<12$ weeks, 10 patients had an Eastern Cooperative Oncology Group Performance Status (ECOG PS) of $\geq 3$ and 64 patients had a history of hepatectomy (8), preoperative chemotherapy (6), prior transarterial chemoembolization (TACE) or local ablation (44), and radiotherapy (6). Consequently, 192 patients were excluded from this study. A total of 104 patients with advanced HCC were eligible for evaluation (Table I). The diagnosis of HCC (6) was indicated by imaging findings and confirmed by biopsy (single action biopsy device, $16 \mathrm{~g}$; Promex Technologies, Franklin, IN, USA). The histological grade of the tumor differentiation was determined by the Edmondson classification into well, moderately and poorly differentiated (22). Portal vein thrombosis (PVT), as a sign of macroscopic vascular invasion and extrahepatic spread, was used to define advanced HCC; however, patients exhibiting extrahepatic spread were excluded from the study. Eligibility criteria also included ECOG PS of 0,1 or 2; Child-Pugh class A or B; life expectancy of at least 12 weeks; total bilirubin concentration of $\leq 51.3 \mu \mathrm{mol} / \mathrm{l}$ and HBV DNA positivity. In addition, patients considered for inclusion were required to exhibit at least one tumor lesion that could be measured along one dimension according to modified RECIST (mRECIST) assessment for HCC (23).

Study design. According to the SHARP trial (13), the overall survival (OS) rate of the advanced HCC patients for sorafenib at the 15 -month time-point was $37 \%$. The sample size calcula- tion is based on the detection of significant differences in OS (the second end-point parameter of this trial), assuming that the OS rate is $50 \%$ for the combination therapy group at the 15-month interval. A total of 90 patients were required for a log-rank test with an overall two-sided significance level of 0.05 and power of 0.805 . From our experience, it was expected that $15-20 \%$ of the patients would drop-out following randomization. In order to accommodate for the drop-out rate, the total sample size was thereby increased to 104 . Study randomization was centralized, investigator-initiated and was approved by the Ethics Committee of the Beijing 302nd Hospital (China). Written informed consent was obtained from the patients prior to enrollment. All eligible patients were randomly assigned (with a $1: 1$ ratio) to the sorafenib and cryoRx group $(n=52)$ or the sorafenib-alone group $(n=52)$ using simple computer randomization to achieve a balance between the two groups. All eligible patients received continuous oral treatment with $0.1 \mathrm{~g}$ of lamivudine once daily. None of the patients had received prior treatment, such as chemotherapy or radiation therapy.

\section{Treatment and disease assessment}

Sorafenib administration. All patients received sorafenib at a dose of $400 \mathrm{mg}$ twice daily for at least 8 weeks. Treatment interruptions and dose reductions (first $400 \mathrm{mg}$ twice daily, then $200 \mathrm{mg}$ twice daily) were permitted for adverse drug reactions (ADRs) according to the National Cancer Institute Common Toxicity Criteria (24). For ADRs of grade 3-4, the sorafenib dose was decreased to $200 \mathrm{mg}$ twice daily until the ADRs improved to a grade of $\leq 2$, then increased to $400 \mathrm{mg}$ twice daily if well tolerated. Therapy was discontinued if the following criteria were met: ADRs that required termination of medication, deterioration of ECOG PS score to 4 and withdrawal of consent. If disease progression was observed, sorafenib was continued when the patient was considered to have a good clinical status (e.g., PS, liver function and tolerable side-effects) and wished to continue the treatment. Following sorafenib treatment, cryoRx was conducted in those without absolute contraindications, based on the potential clinical benefits expected from the treatment and the patient's consent. Sorafenib therapy was continued without interruption during local therapies.

CryoRx procedure. Cryoablation was performed as described previously (25). Briefly, the size and number of the probes depended on the location and average size of the lesions to be ablated. An argon-helium gas-based CRYOcare system (EndoCare, Inc., Irvine, CA, USA) and cryoprobes (2 and $3 \mathrm{~mm}$ ) were used to freeze the tumor with a dual freezethaw cycle under ultrasound-guidance. For optimum reduction of tumor burden, cryoablation was carried out in single or repeated doses. For tumors $<5 \mathrm{~cm}$ in diameter, the aim was for complete ablation, for larger tumors, the aim was to reduce the tumor burden by at least $60 \%$ of the original tumor burden. Cryoablation was limited to three procedures at most.

Immunohistochemical staining for CD34. All samples from HCC patients were reviewed histologically using hematoxylin and eosin staining, the paraffin-embedded samples were cut into $5-\mu \mathrm{m}$ sections and processed for immunohistochemistry according to the manufacturer's instructions, as previously described (26). Tumor sections were immunostained with human CD34 monoclonal antibody (BioGenex, San Ramon, 
Table I. Demographic and baseline characteristics of patients.

\begin{tabular}{|c|c|c|}
\hline Variable & $\begin{array}{l}\text { Combination therapy } \\
\qquad(\mathrm{n}=52)\end{array}$ & Sorafenib $(n=52)$ \\
\hline Age (years) & $51.2 \pm 11.9$ & $52.6 \pm 8.3$ \\
\hline \multicolumn{3}{|l|}{ Gender (\%) } \\
\hline Male & $48(92.3)$ & $47(90.4)$ \\
\hline Female & $4(7.7)$ & $5(9.6)$ \\
\hline \multicolumn{3}{|l|}{ ECOG performance status (\%) } \\
\hline 0 & $16(30.8)$ & $17(32.7)$ \\
\hline 1 & $29(55.7)$ & $30(58)$ \\
\hline 2 & $7(13.5)$ & $5(9)$ \\
\hline BCLC stage C (\%) & $52(100)$ & $52(100)$ \\
\hline Tumor diameter - cm (range) & $8.39 \pm 4.38(3.5-12.8)$ & $8.32 \pm 2.72(3.2-13.2)$ \\
\hline \multicolumn{3}{|l|}{ Number of tumor sites (\%) } \\
\hline 1 & $7(13.5)$ & $6(11.5)$ \\
\hline 2 & $9(17.3)$ & $10(19.2)$ \\
\hline 3 & $10(19.2)$ & $11(21.2)$ \\
\hline$\geq 4$ & $26(50)$ & $25(48.1)$ \\
\hline \multicolumn{3}{|c|}{ Macroscopic vascular invasion (\%) } \\
\hline Branch & $36(69.3)$ & $37(71.2)$ \\
\hline Trunk & $16(30.7)$ & $15(28.8)$ \\
\hline \multicolumn{3}{|l|}{ Differentiated tumor (\%) } \\
\hline Well & $9(17.3)$ & $10(19.2)$ \\
\hline Moderately & $30(57.7)$ & $30(57.7)$ \\
\hline Poorly & $13(25)$ & $12(23.1)$ \\
\hline \multicolumn{3}{|l|}{ HBV DNA positive (\%) } \\
\hline $100-9,999$ & $22(42.3)$ & $24(46.2)$ \\
\hline $10,000-99,999$ & $19(36.5)$ & $18(34.6)$ \\
\hline$\geq 100,000$ & $11(21.2)$ & $10(19.2)$ \\
\hline \multicolumn{3}{|l|}{ Child-Pugh class (\%) } \\
\hline A & $41(78.8)$ & $43(83)$ \\
\hline $\mathrm{B}$ & $11(21.2)$ & $9(17)$ \\
\hline
\end{tabular}

HBV, hepatitis B virus; ECOG, Eastern Cooperative Oncology Group; BCLC, Barcelona Clinic Liver Cancer.

CA, USA). Tissue sections were incubated with primary CD34 monoclonal antibodies. Subsequently, secondary biotinylated antimouse immunoglobulin (Dako North America, Inc., USA) was applied and reacted with streptavidin biotinylated horseradish peroxidase complex (Dako). The negative control was obtained by substituting the primary antibodies with mouse immunoglobulin G.

Determination of MVD. The intratumoral MVD was evaluated by two independent observers who were blinded to the patients' clinical data. The tissue sections were screened at a low power field (x40) and the five areas with the most intense neovascularization (hot spots) were selected. Microvessel counts of these areas were performed with a high power field (x200). To reduce observer-related variation, counting of microvessels was performed using the computer image analyzer (MetaMorph Imaging System Version 3.0; Universal Imaging Corp, West
Chester, PA, USA). Microvessels, tumor cells and connective elements were counted as one microvessel, irrespective of the presence of a vessel lumen. The mean microvessel count of the five most vascular areas was taken to constitute the MVD, which was expressed as the absolute number of microvessels per $0.74 \mathrm{~mm}^{2}$ (x200 field).

Disease assessment. Based on the computed tomography/ magnetic resonance tomography (MRT) scans of the liver performed at baseline and every 8 weeks, tumor response (sorafenib combined with cryoRx or sorafenib alone) was evaluated according to mRECIST (22) assessment for HCC by independent radiologists. Patients who succumbed to the disease prior to their first radiographic control were judged as having progressive disease (PD). Patients who exhibited complete response (CR) or partial response (PR) were defined as achieving a clinical efficacy response (CER). 


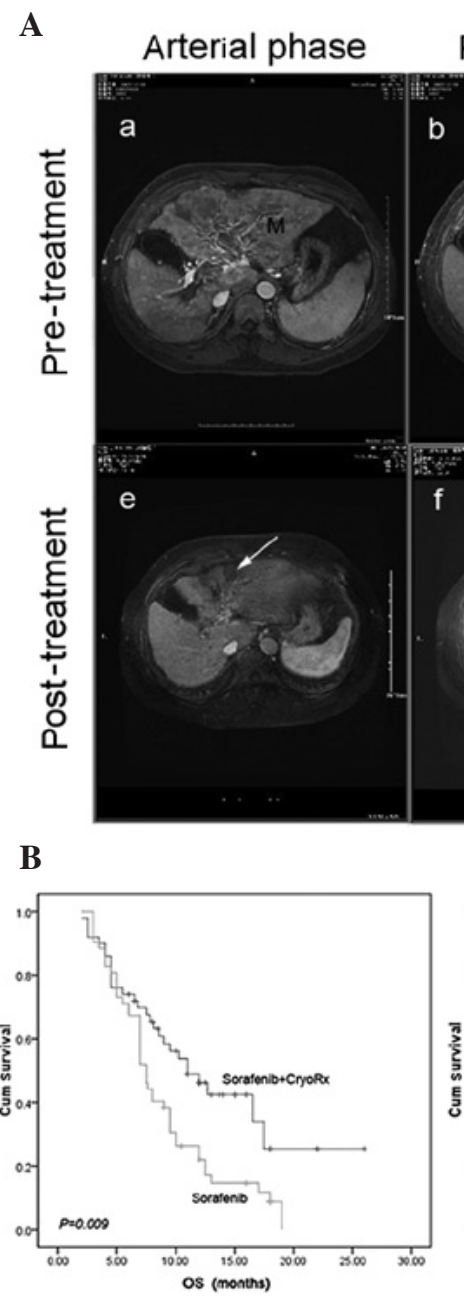

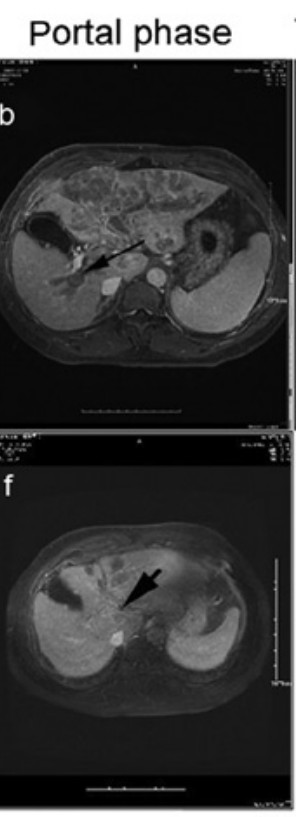

C

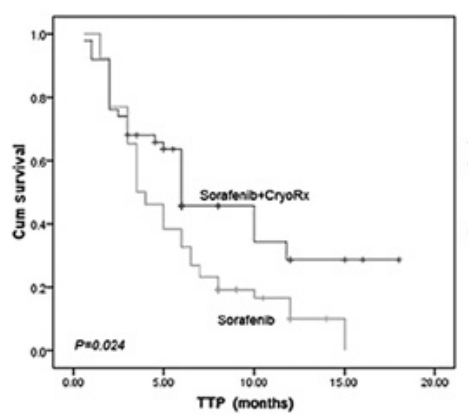

T2-weighted image Coronal image

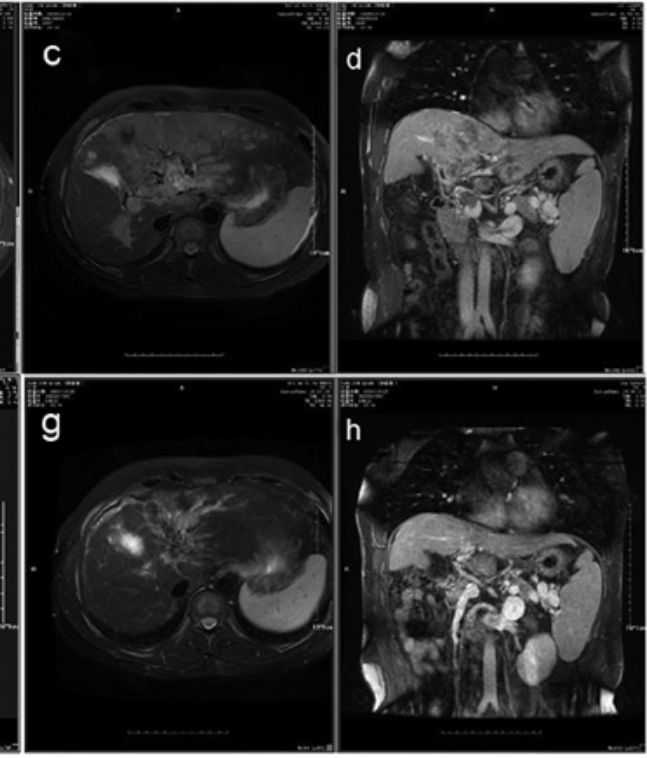

D

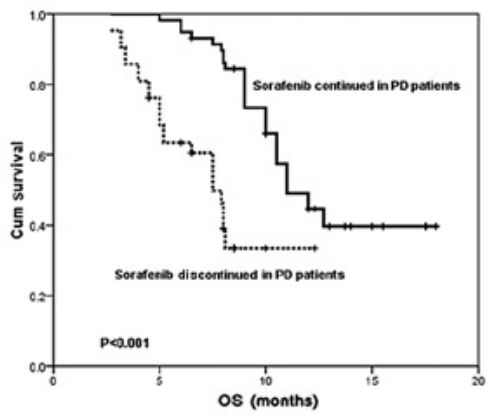

Figure 1. Overall response and efficacy of the combined sorafenib and cryotherapy in advanced HCC. (A) Overall response was a complete response in a live 55-year-old male over a 24-month period at end-point. (A-a) MRI scan reveals a huge mass (M) at the dome of the left hepatic lobe (upper section). (A-b) Long black arrow indicates portal vein thrombosis, histopathological diagnosis was HCC, Edmondson classification to moderately differentiated. (A-c) The large mass at the dome of the left hepatic lobe is shown on T2-weighted image of MRI. (A-d) Portal vein thrombosis is shown on coronal image of MRI. Following continuation of sorafenib treatment, concurrent receipt of 2-times percutaneous cryoablation reduced the tumor burden up to $60 \%$ of the original tumor burden 2 weeks, 10 months later. Not only did the treated tumor shrink, but the non-treated tumor also decreased (lower). (A-e) Long white arrow reveals that the treated tumor was necrotic. (A-f) Short black arrow indicates that the portal vein tumor thrombus was almost invisible. (A-g) T2-weighted image of MRI shows the treated tumor. (A-h) Coronal image of MRI indicates that the portal vein tumor thrombus was almost invisible. (B) Kaplan-Meier survival curves for 52 patients with the combination therapy and 52 patients with sorafenib-alone treatment; the median OS was significantly different between groups. (C) Kaplan-Meier survival curves displaying significant differences in TTP in patients between the combination therapy and sorafenib-alone treatment groups. (D) Kaplan-Meier analysis for the effect of continuing sorafenib therapy in patients with radiologic progressive disease on OS. TTP, time to progression; OS, overall survival; HCC, hepatocellular carcinoma; MRI, magnetic resonance imaging; PD, progressive disease; Cum, cumulative.

End-points. The primary end-point of the study was OS. The secondary end-points included time to progression (TTP), the disease-control rate (DCR) and tolerability. The OS was defined as the time from sorafenib initiation to the date of expiration or the patient's last follow-up. TTP was defined as the time from sorafenib initiation to the date of disease progression or expiration. The disease progression was defined as the tumor progression according to mRECIST criteria or progression of cirrhosis.

Statistical analysis. Continuous data were expressed as the median and range. All continuous data were classified into subgroups according to the median for analysis. Associations between OS, TTP and potential prognostic factors were assessed by the Kaplan-Meier method (log-rank test) in a univariable analysis. The Cox proportional hazards model was used for multivariate analyses with a step-wise procedure and a significance level of 0.10 was used to enter and remove variables. All statistical analyses were performed using SPSS software version 16.0 for Windows (SPSS Inc., Chicago, IL, USA). P-values $<0.05$ were considered to indicate statistically significant differences.

\section{Results}

Patient characteristics. The combination therapy and sorafenib-alone treatment groups were well balanced with regard to baseline demographic and disease characteristics (Table I). A total of $84(80.8 \%)$ patients were classified as Child-Pugh class A and 20 (19.2\%) patients were classified as Child-Pugh class B. A total of $33(31.7 \%)$ patients were ECOG PS 0,59 patients $(56.7 \%)$ were ECOG PS 1 , and 12 patients 
Table II. Summary of efficacy measures.

\begin{tabular}{|c|c|c|c|}
\hline Outcome & Combination therapy $(\mathrm{n}=52)$ & Sorafenib $(\mathrm{n}=52)$ & P-value \\
\hline Overall survival (months) & 0.009 & & \\
\hline Median & 12.5 & 8.6 & \\
\hline$(95 \% \mathrm{CI})$ & $10.6-16.4$ & 7.3-10.4 & \\
\hline Time to progression & 0.024 & & \\
\hline Median & 9.5 & 5.3 & \\
\hline$(95 \% \mathrm{CI})$ & $8.4-13.5$ & $3.8-6.9$ & \\
\hline \multicolumn{4}{|l|}{ Level of response $(\%)$} \\
\hline Complete response & $3(5.7)$ & 0 & NA \\
\hline Partial response & $9(17.3)$ & $4(7.6)$ & 0.1186 \\
\hline Stable disease & $22(42.3)$ & $19(36.5)$ & 0.4423 \\
\hline $\operatorname{CER}(\%)$ & $12(23.0)$ & $4(7.6)$ & 0.0314 \\
\hline DCR $(\%)$ & $34(65.4)$ & $23(44.2)$ & 0.0212 \\
\hline
\end{tabular}

CER, clinical efficacy rate (defined as the proportion of patients who had a best response rating of complete response, partial response, which was maintained $\geq 4$ weeks from the first manifestation of that rating). DCR, disease control rate (defined as the proportion of patients who had a best response rating of complete response, partial response or stable disease, which was maintained $\geq 4$ weeks from the first manifestation of that rating). CI, confidence interval.

(11.6\%) were ECOG PS 2. The tumor differentiation was high in $19(18.3 \%)$, intermediate in $60(57.7 \%)$ and low in 25 patients (24.0\%). The HBV DNA loads were low in 46 (44.2\%), intermediate in $37(35.6 \%)$ and high in 21 patients $(20.2 \%)$.

Adverse events. With regard to non-hematological toxicity, rash was observed most commonly (62\%), followed by hypertension (56\%), weight loss $(52.9 \%)$, alopecia $(50 \%)$, diarrhea (46\%), fatigue $(43.3 \%)$, hand-foot skin reaction (HFSR) $(42 \%)$, liver dysfunction (34.6\%), voice change (18\%), abdominal pain $(12.5 \%)$ and upper gastrointestinal tract bleeding $(16 \%)$. Furthermore, the grade 3 or 4 non-hematological toxicities were HFSR, diarrhea, liver dysfunction and upper gastrointestinal tract bleeding, which occurred in 12.2, 12, 6.4 and $6 \%$ of patients, respectively. With regard to hematological toxicity, leukopenia (24\%) was the most common sign of toxicity, followed by thrombocytopenia (12\%) and anemia (8\%).

Overall response and efficacy. The median time of follow-up was 10.5 months (range 4.0-26.0) and the median duration of sorafenib treatment was 7.5 months (2.5-26.0). A total of 10 (9.6\%) patients discontinued sorafenib treatment at 6-24 weeks due to liver function deterioration (6 cases) and bleeding from gastroesophageal varices (4 cases), 21 (20.2\%) patients reduced sorafenib dosage to $200 \mathrm{mg}$ twice daily due to grade 3-4 ADRs. However, all these patients restored the dose to $400 \mathrm{mg}$ twice daily after 2 to 3 weeks. Overall, patients receiving the combination therapy had a median OS of 12.5 months (95\% CI 10.6-16.4), compared to 8.6 months (95\% CI 7.3-10.4) for those receiving sorafenib (log-rank, $\mathrm{P}=0.009$; Fig. 1B). In addition, patients receiving combination therapy had a significantly longer median TTP [9.5 months (95\% CI 8.4-13.5)] than patients receiving sorafeinb [5.3 months (95\% CI 3.8-6.9), log-rank $\mathrm{P}=0.024$; Fig. 1C). In the analysis for best response, 4 out of 52 patients receiving combination therapy $(7.6 \%$, Fig. 1A) exhibited a CR, 9 patients $(17.3 \%)$ exhibited a PR, 22 patients (42.3\%) exhibited stable disease (SD), whereas out of the patients receiving sorafenib treatment alone, $4(7.6 \%)$ and $19(36.5 \%)$ patients exhibited PR and SD, respectively. The rates of CER and DCR were significantly higher in the combination therapy group (CER, 22\% and DCR, 66\%) than in the sorafenib group [CER, 7.6\% $(\mathrm{P}=0.04)$ and DCR, $44.2 \%(\mathrm{P}=0.03)$; Table II]. A total of 67 advanced HCC patients receiving lamivudine treatment with HBV DNA-negative status had a median OS of 11.5 months (95\% CI 6.3-14.8), compared to 8.0 months $(95 \%$ CI 5.8-11.5) for the 37 patients receiving lamivudine treatment with HBV DNA-positive status (log-rank, $\mathrm{P}=0.035)$. There were no significant differences between the median TTP for the two groups (log-rank, $\mathrm{P}=0.544)$. Disease progression occurred in $86(82.6 \%)$ patients, the OS was significantly longer in 50 patients with a clinically stable presentation who continued sorafenib treatment than in those with clinical deterioration who discontinued therapy (11 vs. 7.5 months, $\mathrm{P}<0.001$; Fig. 1D). Furthermore, 53 (50.9\%) patients succumbed to the disease due to recurrence/metastasis in 25 (24\%), liver failure in $13(12.5 \%)$, bleeding from gastroesophageal varices in $8(7.7 \%)$, refractory ascites-induced renal failure in $4(3.8 \%)$ and tumor rupture/hemorrhage in 3 patients $(2.9 \%)$.

Response and efficacy according to intratumoral MVD. Specific staining of capillary-like vessels by anti-CD34 was observed in intratumoral specimens in all outcome groups $(\mathrm{CR}+\mathrm{PR}$, mean MVD-CD34, 111 $\pm 49 / 0.74 \mathrm{~mm}^{2}$; SD, 206 $\pm 74 / 0.74 \mathrm{~mm}^{2}$; PD, $339 \pm 92 / 0.74 \mathrm{~mm}^{2}$; Fig. 2A and B). The mean MVD-CD34 in the responsive $(\mathrm{CR}+\mathrm{PR})$ patients was significantly lower than that of PD patients $(\mathrm{P}<0.001)$. At the time of analysis, all patients were divided into two groups by the median MVD value (median, 219.5/0.74 $\mathrm{mm}^{2}$; range, from 34 to $512 / 0.74 \mathrm{~mm}^{2}$ ). When the entire cohort of 104 patients was analyzed, 53 patients with low intratumoral MVD $\left(\leq 219.5 / 0.74 \mathrm{~mm}^{2}\right)$, receiving combination therapy, had longer TTP and OS than that of patients receiving 
A

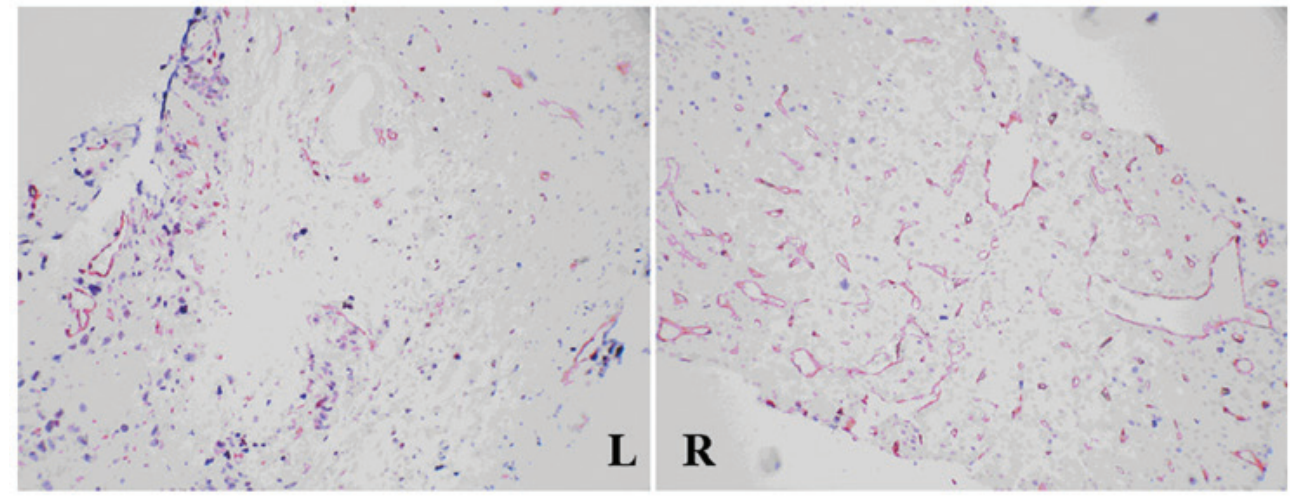

B

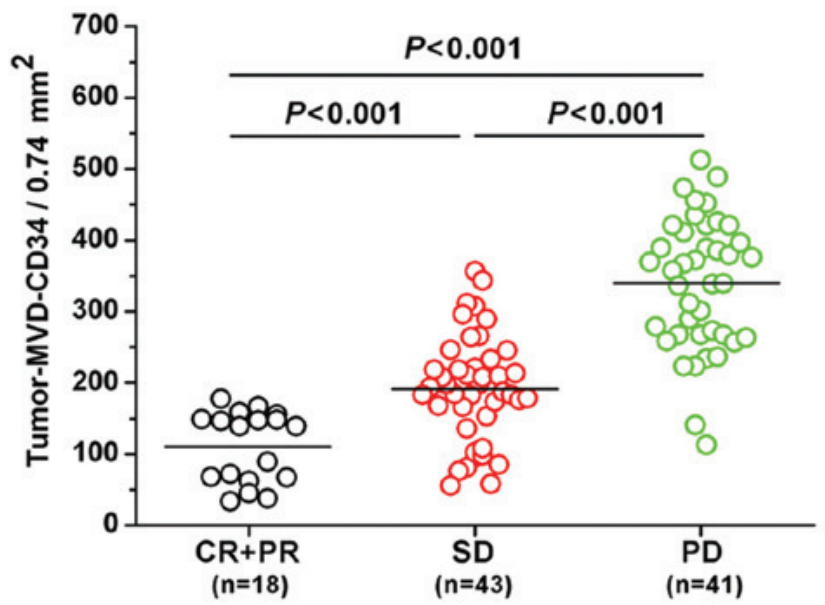

C
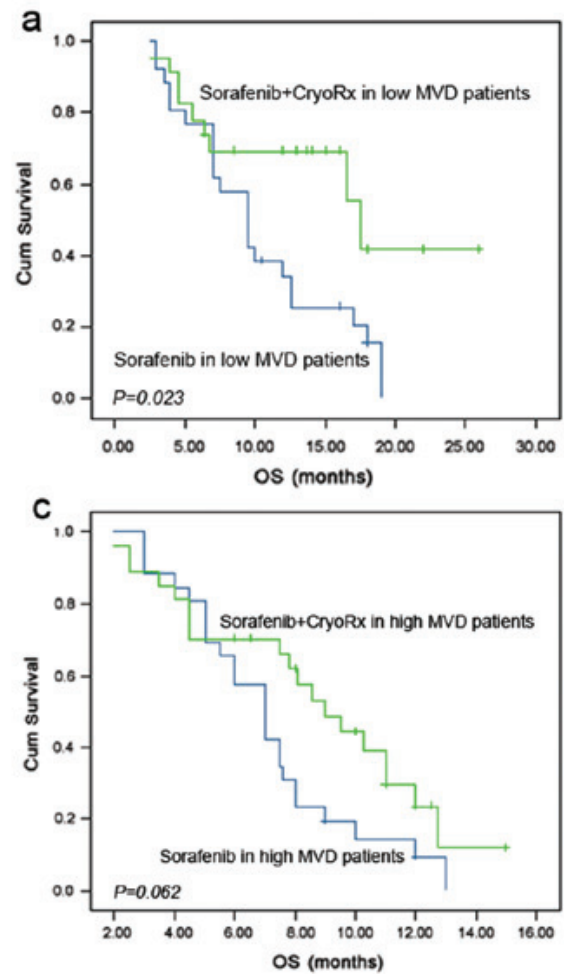

b
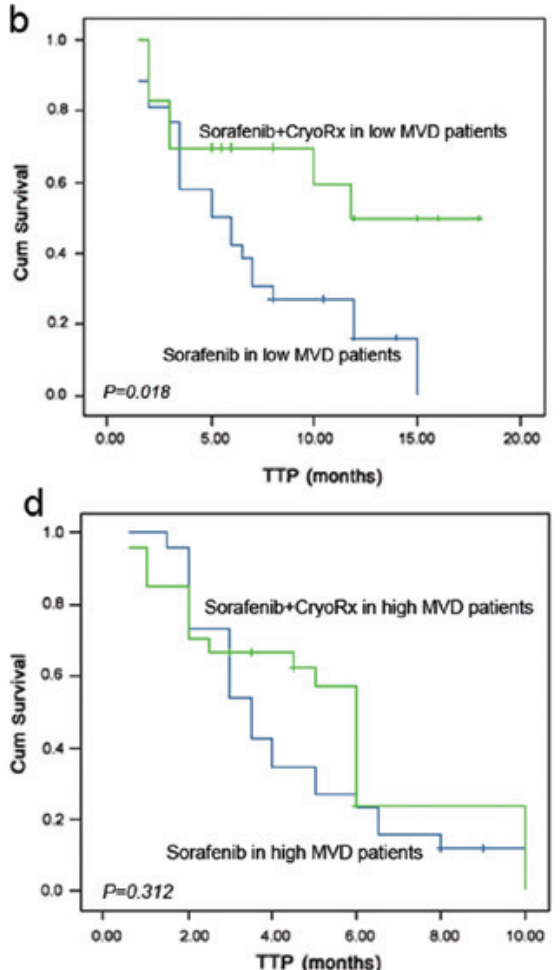

Figure 2. Comparison of intratumoral MVD-CD34 in advanced HCC patients displaying various overall responses. (A) Intratumoral MVD by anti-CD34 immunostaining (pink staining) (left, low MVD-CD34; right, high MVD-CD34; x200). (B) The mean intratumoral MVD-CD34 significantly increased with poor overall response. (C) Analysis of a cohort of 104 patients. Kaplan-Meier survival curves for 51 patients with low MVD-CD34 ( $\left.\leq 219.5 / 0.74 \mathrm{~mm}{ }^{2}\right) ;(\mathrm{C}-\mathrm{a})$ compared to the TTP, revealed significant differences in patients between the combination therapy and sorafenib-alone treatment groups; (C-b) the median OS was significantly different between groups. Kaplan-Meier survival curves for 53 patients with high MVD-CD34 (>219.5/0.74 mm²); (C-c) the TTP and (C-d) the OS displayed no significant differences between the two groups. OS, overall survival; PD, progressive disease; SD, stable disease; CR, complete response; $\mathrm{PR}$, partial response; MVD, microvessel density; TTP, time to progression; Cum, cumulative. 
Table III. Univariate analysis of patient demographics and clinical characteristics for predictive factors of TTP and OS.

\begin{tabular}{|c|c|c|c|c|c|}
\hline \multirow[b]{2}{*}{ Parameter } & \multirow{2}{*}{$\begin{array}{l}\text { No. of patients } \\
\text { who expired }\end{array}$} & \multicolumn{2}{|c|}{ TTP (months) } & \multicolumn{2}{|c|}{ OS (months) } \\
\hline & & Median & P-value & Median & P-value \\
\hline Gender & & & 0.514 & & 0.781 \\
\hline Male & 48 & 6.0 & & 10.5 & \\
\hline Female & 5 & 4.5 & & 9.0 & \\
\hline Age (years) & & & 0.668 & & 0.228 \\
\hline$\leq 51$ & 28 & 5.0 & & 9.0 & \\
\hline$>51$ & 25 & 6.0 & & 10.5 & \\
\hline ECOG PS & & & $<0.001$ & & $<0.001$ \\
\hline 0 & 7 & 8.5 & & 17.0 & \\
\hline 1 & 35 & 6.0 & & 11.0 & \\
\hline 2 & 11 & 3.0 & & 6.5 & \\
\hline Tumor differentiation & & & 0.155 & & 0.401 \\
\hline Well & 10 & 4.5 & & 9.0 & \\
\hline Moderate & 29 & 4.0 & & 8.0 & \\
\hline Poor & 14 & 3.0 & & 7.0 & \\
\hline Tumor diameter $(\mathrm{cm})$ & & & 0.025 & & 0.007 \\
\hline$\leq 7$ & 19 & 6.0 & & 12.0 & \\
\hline$>7$ & 34 & 5.0 & & 8.1 & \\
\hline Tumor number & & & 0.165 & & 0.995 \\
\hline 1 & 15 & 6.0 & & 12.7 & \\
\hline 2 & 11 & 6.0 & & 11.0 & \\
\hline 3 & 11 & 5.0 & & 10.0 & \\
\hline 4 & 21 & 4.0 & & 10.0 & \\
\hline Invasion of portal vein & & & 0.019 & & 0.040 \\
\hline Branch & 30 & 6.0 & & 11.0 & \\
\hline Trunk & 23 & 4.0 & & 9.0 & \\
\hline HBV DNA (IU/ml) & & & $<0.001$ & & $<0.001$ \\
\hline $0-9,999$ & 26 & 6.0 & & 12.7 & \\
\hline $10,000-99,999$ & 10 & 4.0 & & 10.0 & \\
\hline$\geq 100,000$ & 22 & 3.0 & & 8.0 & \\
\hline Therapy & & & $<0.001$ & & $<0.001$ \\
\hline Combined treatment & 21 & 9.5 & & 12.5 & \\
\hline Sorafenib alone & 32 & 5.3 & & 8.6 & \\
\hline Child-Pugh class & & & $<0.001$ & & $<0.001$ \\
\hline $\mathrm{A}$ & 39 & 6.0 & & 11.5 & \\
\hline $\mathrm{B}$ & 14 & 3.5 & & 7.0 & \\
\hline Weight loss & & & 0.001 & & $<0.001$ \\
\hline Grade 0 & 13 & 6.5 & & 11.2 & \\
\hline Grade 1-4 & 40 & 4.0 & & 7.0 & \\
\hline Fatigue & & & $<0.001$ & & $<0.001$ \\
\hline Grade 0 & 21 & 7.0 & & 13.0 & \\
\hline Grade 1-4 & 32 & 4.0 & & 8.1 & \\
\hline AFP levels (ng/ml) & & & 0.005 & & 0.001 \\
\hline$\leq 895$ & 20 & 6.0 & & 12.7 & \\
\hline$>895$ & 33 & 4.0 & & 9.0 & \\
\hline Intratumoral MVD & & & $<0.001$ & & $<0.001$ \\
\hline High & 34 & 3.0 & & 7.0 & \\
\hline Low & 19 & 8.8 & & 13.5 & \\
\hline
\end{tabular}

PFS, progress-free survival; OS, overall survival; ECOG, Eastern Cooperative Oncology Group; PS, performance status; HBV, hepatitis B virus; AFP, $\alpha$-fetoprotein; TTP, time to progression; MVD, microvessel density. 
Table IV. Multivariable analysis of predictive factors for TTP and OS by Cox proportional hazards model.

\begin{tabular}{lll}
\hline Variable & $\begin{array}{l}\text { Risk ratio } \\
(95 \% \mathrm{CI})\end{array}$ & P-value \\
\end{tabular}

TTP

$\begin{array}{llr}\text { ECOG PS } & 5.213(3.052-9.309) & <0.001 \\ \text { Combined treatment } & 0.576(0.399-0.831) & 0.003 \\ \text { Child-Pugh class } & 2.628(1.416-4.878) & 0.002 \\ \text { Low MVD } & 1.626(0.604-3.516) & 0.006 \\ \text { OS } & & \\ \text { ECOG PS } & 8.689(4.315-18.396) & <0.001 \\ \text { Combined treatment } & 0.245(0.071-0.846) & 0.026 \\ \text { AFP } & 2.260(1.174-4.352) & 0.015 \\ \text { Low MVD } & 4.618(3.213-11.236) & <0.001\end{array}$

ECOG, Eastern Cooperative Oncology Group; PS, performance status; HBV, hepatitis B virus; AFP, $\alpha$-fetoprotein; MVD, microvessel density; TTP, time to progression; OS, overall survival; CI, confidence interval.

sorafenib-alone treatment [log-rank, $\mathrm{P}=0.018$ (TTP); $\mathrm{P}=0.023$ (OS); Fig. 2C-a and b]. TTP and OS were not significantly different between groups [log-rank, $\mathrm{P}=0.312$ (TTP); $\mathrm{P}=0.062$ (OS); Fig. 2C-c and d] in 51 patients exhibiting a high intratumoral MVD $\left(>219.5 / 0.74 \mathrm{~mm}^{2}\right)$.

Univariate and multivariate analysis of predictive factors for TTP and OS. The combination therapy or the sorafenib-alone treatment groups exhibited a clinical benefit in all preplanned subgroup analyses, despite certain patients having characteristics associated with poor prognosis, including poorer ECOG PS $\geq 1$, tumor diameter $>7 \mathrm{~cm}$, PVT within the trunk, high HBV DNA load, Child-Pugh class B, fatigue, weight loss, $\alpha$-fetoprotein (AFP) levels of $>895 \mathrm{ng} / \mathrm{ml}$ and high intratumoral MVD (Table III).

Cox proportional hazards model analyses (TableIV) revealed that cryoRx was independently associated with improved TTP and OS of sorafenib treatment for advanced HCC. High intratumoral MVD was independently associated with poor clinical outcome of sorafenib for treatment of advanced HCC. Whereas poor ECOG PS (OR 5.213, 95\% CI 3.052-9.309; P<0.001) and high Child-Pugh class (OR 2.628, 95\% CI 1.416-4.878; $\mathrm{P}=0.002$ ) were independently associated with worse TTP. Moreover, good ECOG PS (OR 8.698, 95\% CI 4.315-18.396; $\mathrm{P}<0.001)$ and low AFP levels (OR 2.260, 95\% CI 1.174-4.352; $\mathrm{P}=0.015$ ) were independently associated with better OS.

\section{Discussion}

To the best of our knowledge, this report is the first to describe cryoRx as being associated with improved clinical outcomes of sorafenib treatment for advanced HCC. Sorafenib demonstrated a significant survival benefit and high tolerance in patients with advanced HCC in a phase III clinical trial (13), and was simultaneously found to impede tumor burden limit in advanced HCC (27). It is crucial to reduce tumor burden in order to increase the clinical responses of drugs (28). CryoRx has been proposed as a valid alternative to surgery for the treatment of HCC in patients with cirrhosis (29). Recent studies have examined the outcomes of percutaneous cryoRx for HCC using CT monitoring and MR guidance, reporting that it is safe and efficacious (30). Moreover, it was found that not only was the local tumor necrotic, but also that the adjacent tumor tissue was necrotic and shrunken in HCC patients following cryoRx, which was regarded as reflecting ectopic tumor suppression (16). We report that following cryoablation for small HCC the 1-, 2- and 3-year recurrence-free survival rates were 72, 56 and $43 \%$, respectively (25). As such, local recurrence following cryoRx represents one of the major problems of this therapy, and limits its associated survival benefits. We hope that the combinaion of sorafenib and cryoRx may be used to overcome tumor burdens and local recurrence for advanced HCC patients, and provide significant survival benefits.

In the present study, the median OS time of the combination therapy was 12.5 months, significantly longer than that of patients receiving the sorafenib-alone therapy (8.6 months). In addition, the combination therapy significantly prolonged TTP and CER or DCR compared with the sorafenib-alone therapy. The significant improvements in OS and TTP in the combination therapy group provide encouraging evidence that combination therapy may overcome the tumor burden and local tumor recurrence. The OS we found is longer than that reported in all previous studies of sorafenib treatment for advanced HCC patients (12,13,31-33). All patients in our study suffered from advanced HCC (100\% BCLC stage C and HBV DNA-positive) with macroscopic vascular invasion. In $50 \%$ of our patients, the largest tumor diameter was $>7 \mathrm{~cm}$; these characteristics suggest that the patients enrolled in our study may have had greater tumor burden than those studied in previously reported trials $(12,13)$. In the Asian-Pacific study, the median OS and TTP were 6.5 and 2.8 months, respectively, and the population displayed poorer performance (74\% ECOG PS $\geq 1$ ) and a more advanced stage of cancer (96\% BCLC stage C) (12). In the current study, 35 out of 52 patients (67\%) in the sorafenib-alone treatment group exhibited ECOG PS $\geq 1$, all received continuous oral treatment with $0.1 \mathrm{~g}$ of lamivudine once daily, advanced HCC patients with antiviral therapy had the opportunity for sorafenib maintenance therapy by improving liver function. Thus, the sorafenib-alone treatment group had a much better OS and TTP than those reported in the Asian-Pacific trial of sorafenib (12). In the SHARP study (13), the median OS and TTP were 10.7 and 4.1 months, respectively, and the population exhibited a more advanced stage of cancer (82\% BCLC stage C; $38 \%$ macroscopic vascular invasion and $51 \%$ extrahepatic spread). The 12.5 -month OS and 8.5-month TTP observed in patients with PVT in the combination therapy treatment groups are particularly impressive, in accordance with the rationale for the combination treatments by previous findings. Although we found that sorafenib was capable of prolonging survival in advanced HCC patients, monotherapy of sorafenib has not yet been found to produce tumor regression in HCC. High tumor burden may render patients refractory to sorafenib (27).

In accordance with the evidence from previous studies, our results suggest that combination therapy has numerous advantages. First, cryoRx may reduce the tumor burden, 
thus increasing the efficacy of sorafenib. Second, sorafenibmediated blockage of the Raf/mitogen-activated protein kinase and VEGFR pathways might enhance the efficacy of local cryoRx. These possibilities are supported by the current data. The clinical benefits of the combination therapy may be largely due to the reduction of tumor burden by cryoRx, in accordance with previous findings (15). More importantly, the addition of cryoRx to sorafenib treatment could further improve OS in these HCC patients. The profile, frequency and degree of sorafenib-related ADRs were comparable to those from previous reports, and cryoRx did not increase the frequency and degree of sorafenib-related ADRs. These encouraging results indicate that sorafenib combined with cryoRx may provide the best therapeutic benefit in patients suffering from advanced HCC.

A crucial difference between the present study and previous studies was the continuous administration of sorafenib, which may have also contributed to the survival benefit we observed. In the SHARP trial a survival time of 5.2 months was reported following disease progression (12). In a Japanese phase I study of sorafenib, despite the median TTP being only 4.9 months, the median OS was relatively long, at 15.6 months (34). The results of Yau et al indicate that even in patients who did not demonstrate any clinical benefits with sorafenib, OS was substantially improved compared with their historical cohort (27). Wörns et al reported that radiological disease stabilization $(\mathrm{PR}+\mathrm{SD})$ was achieved in $50 \%$ of patients following a median of 3.2 months, or at least a clinically stable presentation in a subset of patients with radiological PD leading to continuation of therapy (35). These findings suggest that even patients who exhibited radiological tumor progression with sorafenib treatment might obtain a survival benefit from the drug. Therefore, the application of radiological progression criteria would be likely to lead to a discontinuation of sorafenib therapy after 3-4 months in these cases, potentially denying these patients the opportunity to continue to receive clinical benefit and improved OS. We propose that the decision to continue therapy with sorafenib following radiological progression is justified in patients with sustained clinically stable presentation.

Sub-analyses were conducted on the basis of various factors associated with the prognosis of patients with $\mathrm{HCC}$, including age, largest tumor diameter, tumor difference, ECOG PS, Child-Pugh class and HBV DNA load. Our data showed that the combination therapy or the sorafenib-alone treatment groups exhibited a clinical benefit in all preplanned subgroup analyses. However, patients with an ECOG PS of 2, tumor diameter $>7 \mathrm{~cm}$, PVT within the trunk, high HBV DNA load, Child-Pugh class B, AFP levels $>895 \mathrm{ng} / \mathrm{ml}$ and high intratumoral MVD were associated with poor prognosis. We also analyzed the correlation of treatment-related toxicities with prognosis. Similar to the results of Vincenzi et al, the fatigue, weight loss and liver toxicity correlated with poor DCR, TTP and OS to a certain extent (36). In a study by Yau et al, fatigue was observed in $50 \%$ of patients (27). In the present study, $44 \%$ of patients exhibited fatigue. As such, we believe that severe fatigue may be predictive of poor prognosis to a certain extent. Liver toxicity is a key issue during sorafenib and local treatment. Compared to previous reports, adding cryoRx did not further increase frequency and degree of sorafenib-related ADRs. This finding suggests that liver toxicity can be induced by sorafenib.
Sorafenib may induce liver failure not only in Child-Pugh B patients but also in Child-Pugh A patients (37). However, in the present study, the majority of sorafenib-induced liver failure occurred in Child-Pugh B patients.

In previous research regarding intratumoral MVD as a prognostic measure of tumor angiogenesis $(26,38)$, a prospective study found a significant positive correlation between MVD and postoperative recurrence in patients undergoing resection of HCCs $\leq 5 \mathrm{~cm}$ (39). Therefore, we analyzed the correlation between intratumoral MVD and the response to sorafenib therapy. Our results showed that the mean MVD of patients with CER was significantly lower than that of patients with $\mathrm{PD}(\mathrm{P}<0.001)$. The results suggest that intratumoral MVD may affect the clinical response to therapy in advanced HCC patients. Among patients with low intratumoral MVD, we found that those receiving the combination therapy exhibited a significantly longer median TTP and OS than those receiving the sorafenib-alone therapy; however, in patients with high intratumoral MVD, TTP and OS were not significantly different between treatments. The current data indicate that the anti-angiogenic effects of sorafenib for advanced HCC patients with a high intratumoral MVD are mild.

Multivariable analysis revealed that cryoRx was independently associated with improved TTP and OS of sorafenib treatment for advanced HCC, and that poor ECOG PS or high intratumoral MVD predicted poor TTP and OS, which is consistent with the results of previous studies (33). In the current study, none of the patients had an ECOG PS $>2$ or a Child-Pugh class worse than B. Ideally, the tumor control rate increases with the sorafenib dose and the completion of local treatment. Patients with a better PS had the opportunity for sorafenib maintenance therapy and successful local treatment due to the acceptable adverse effects. Certain well-established prognostic predictors, including tumor differentiation and vascular invasion, were not associated with survival. Although tumor size was significantly associated with OS based on the log-rank test, which suggests that tumor burden might be a mechanism involved in refraction to sorafenib, it was excluded from multivariate analyses. Similar to the results of previous studies, Child-Pugh class and AFP were independently associated with TTP and OS, respectively. The precise mechanism by which AFP affects prognosis remains unclear. However, several studies have reported that AFP is a novel protein-binding partner for caspase-3, which blocks the apoptotic signaling pathway, and promotes the growth of human hepatoma cells as a co-repressor in retinoic acid (RA)-RA receptor signaling $(40,41)$. Therefore, the low AFP level was independently associated with better OS.

In conclusion, this clinical study demonstrates that compared to the sorafenib-alone treatment group, the addition of cryoRx to sorafenib treatment significantly improves the clinical outcomes of sorafenib for the treatment of advanced HCC, with acceptable tolerance and similar safety profiles as previously reported. High intratumoral MVD was predictive of poor responses to sorafenib therapy for advanced HCC patients. These results provide further validation for sorafeinb treatment for advanced HCC.

\section{Acknowledgements}

The authors would like to thank all the patients enrolled in this study for their kind understanding and support. The 
present study was supported by a grant from the Key Scientific and Technological Research Foundation of the National Special-purpose Program (No. 2008ZX10002-018) and the Capital Medical Development and Research Fund (2007-1021, 2009-2041).

\section{References}

1. Parkin DM: Global cancer statistics in the year 2000. Lancet Oncol 2: 533-43, 2001.

2. Tang ZY: Perspective of clinical onology from the viewpoint of liver cancer studies. Tumor (China) 29: 1-4, 2009.

3. Yoo HY, Patt CH, Geschwind JF, et al: The outcome of liver transplantation in patients with hepatocellular carcinoma in the United States between 1988 and 2001: 5-year survival has improved significantly with time. J Clin Oncol 21: 4329-4335, 2003.

4. Yang HI, Lu SN, Liaw YF, You SL, Sun CA, Wang LY, Hsiao CK, Chen PJ, Chen DS and Chen CJ: Hepatitis B e antigen and the risk of hepatocellular carcinoma. N Engl J Med 347: 168-174, 2002.

5. Sherman M: Hepatocellular carcinoma: epidemiology, risk factors, and screening. Semin Liver Dis 25: 143-154, 2005.

6. Llovet JM, Burroughs A and Bruix J: Hepatocellular carcinoma. Lancet 362: 1907-1917, 2003.

7. Llovet JM, Burroughs A and Bruix J: Prognosis of hepatocellular carcinoma: the BCLC staging classification. Semin Liver Dis 19: 329-338, 1999.

8. Bruix J and Llovet JM: Prognosis prediction and treatment strategy in hepatocellular carcinoma. Hepatology 35: 519-524, 2002.

9. Bruix J and Sherman M: Management of hepatocellular carcinoma. Hepatology 42: 1208-1236, 2005.

10. Park KW, Park JW, Choi JI, et al: Survival analysis of 904 patients with hepatocellular carcinoma in a hepatitis B virusendemic area. J Gastroenterol Hepatol 23: 467-473, 2008.

11. Llovet JM, Fuster J, Bruix J and the Barcelona-Clinic Liver Cancer Group: The Barcelona approach: diagnosis, staging, and treatment of hepatocellular carcinoma. Liver Transpl 10 (Suppl 1): 115-120, 2004.

12. Cheng AL, Kang YK, Chen Z, et al: Randomized phase III trial of sorafenib versus placebo in Asian patients with advanced hepatocellular carcinoma. Lancet Oncol 10: 25-34, 2009.

13. Llovet JM, Ricci S, Mazzaferro V, et al; SHARP Investigators Study Group: Sorafenib in advanced hepatocellular carcinoma. N Engl J Med. 359: 378-390, 2008.

14. Furuse J: Sorafenib for the treatment of unresectable hepatocellular carcinoma. Biologics 2: 779-788, 2008.

15. Hakimé A, Hines-Peralta A, Peddi $\mathrm{H}$, et al: Combination of radiofrequency ablation with antiangiogenic therapy for tumor ablation efficacy: study in mice. Radiology 244: 464-470, 2007.

16. Osada S, Imai H, Tomita H, et al: Serum cytokine levels in response to hepatic cryoablation. J Surg Oncol 95: 491-498, 2007.

17. Poston G: Cryosurgery for colorectal liver metastases. Hepatogastroenterol 48: 323-324, 2001.

18. Lee FT Jr, Mahvi DM, Chosy SG, Onik GM, Wong WS, Littrup PJ and Scanlan KA: Hepatic cryosurgery with intraoperative US guidance. Radiology 202: 624-632, 1997.

19. Shock SA, Laeseke PF, Sampson LA, Lewis WD, Winter TC III, Fine JP and LEE FT Jr: Hepatic hemorrhage caused by percutaneous tumor ablation: radiofrequency ablation versus cryoablation in a porcine model. Radiology 236: 125-131, 2005.

20. Atwell TD, Farrell MA, Callstrom MR, Charboneau JW, Leibovich BC, Patterson DE, Chow GK and Blute ML: Percutaneous cryoablation of 40 solid renal tumors with US guidance and CT monitoring: initial experience. Radiology 243: 276-83, 2007.

21. Jungraithmayr W, Burger D, Olschewski M and Eggstein S: Cryoablation of malignant liver tumor: results of a single center study. Hepatobiliary Pancreat Dis Int 4: 554-560, 2005.

22. Yamamoto J, Kosuge T, Saiura A, et al: Effectiveness of hepatic resection for early-stage hepatocellular carcinoma in cirrhotic patients: subgroup analysis according to Milan Criteria. Jpn J Clin Ocncol 34: 287-295, 2007.
23. Lencioni R and Llovet JM: Modified RECIST (mRECIST) assessment for hepatocellular carcinoma. Semin Liver Dis 30: 52-60, 2010.

24. Trotti A, Colevas AD, Setser A, et al: CTCAE v 3.0: development of a comprehensive grading system for the adverse effects of cancer treatment. Semin Radiat Oncol 13: 176-181, 2003.

25. Wang C, Lu Y, Chen Y, Feng Y, An L, Wang X, Su S, Bai W, Zhou L, Yang Y and Xu D: Prognostic factors and recurrence of hepatitis B-related hepatocellular carcinoma after argon-helium cryoablation: a prospective study. Clin Exp Metastasis 26: 839-848, 2009.

26. Poon RT, Ng IO, Lau C, Yu WC, Yang ZF, Fan ST and Wong J: Tumor microvessel density as a predictor recurrence after resection of hepatocellular carcinoma: a prospective study. J Clin Oncol 20: 1775-1785, 2002

27. Yau T, Chan P, Ng KK, et al: Phase 2 open-label study of single-agent sorafenib in treating advanced hepatocellular carcinoma in a hepatitis B-endemic Asian population: presence of lung metastasis predicts poor response. Cancer 115: 428-436, 2009.

28. Greten TF, Korangy F, Manns MP and Malek NP: Molecular therapy for the treatment of hepatocellular carcinoma. Br J Cancer 100: 19-23, 2009

29. Zuro LM and Staren ED: Cryosurgical ablation of unresectable hepatic tumors. AORN J 64: 231-6, 239-44, 1996.

30. Shimizu T, Sakuhara Y, Abo D, Hasegawa Y, Kodama Y, Endo H, Shirato $\mathrm{H}$ and Miyasaka K: Outcome of MR-guided percutaneous cryoablation for hepatocellular carcinoma. J Hepatobiliary Pancreat Surg 16: 816-823, 2009.

31. Shim JH, Park JW, Choi JI, Park BJ and Kim CM: Practical efficacy of sorafenib monotherapy for advanced hepatocellular carcnoma patients in a Hepatitis B virus-endemic area. J Cancer Res Clin Oncol 135: 617-625, 2009.

32. Pinter M, Sieghart W, Graziadei I, Vogel W, Maieron A, Königsberg R, Weissmann A, Kornek G, Plank C and Peck-Radosavljevic M: Sorafenib in unresectable hepatocellular carcinoma from mild to advanced stage liver cirrhosis. Oncologist 14: 70-76, 2009

33. Kane RC, Farrell AT, Madabushi R, et al: Sorafenib for the treatment of unresectable hepatocellular carcinoma. Oncologist 14: 95-100, 2009.

34. Furuse J, Ishii H, Nakachi K, et al: Phase I study of sorafenib in Japanese patients with hepatocellular carcinoma. Cancer Sci 99: $159-165,2008$.

35. Wörns MA, Weinmann A, Pfingst K, et al: Safety and efficacy of sorafenib in patients with advanced hepatocellular carcinoma in consideration of concomitant stage of liver cirrhosis. J Clin Gastroenterol 43: 489-495, 2009.

36. Vincenzi B, Santini D, Russo A, et al: Early skin toxicity as a predictive factor for tumor control in hepatocellular carcinoma patients treated with sorafenib. Oncologist 15: 85-92, 2010.

37. Schramm C, Schuch G and Lohse AW: Sorafenib-induced liver failure. Am J Gastroenterol 103: 2162-2163, 2008.

38. Semela D and Dufour JF: Angiogenesis and hepatocellular carcinoma. J Hepatol 41: 864-880, 2004.

39. Liu L, Cao Y, Chen C, et al: Sorafenib blocks the RAF/MEK/ ERK pathway, inhibits tumor angiogenesis, and induces tumor cell apoptosis in hepatocellular carcinoma model PLC/PRF/5. Cancer Res 66: 11851-11858, 2006.

40. Li M, Li H, Li C, Guo L, Liu H, Zhou S, Liu X, Chen Z, Shi S, Wei J, McNutt MA and Li G: Cytoplasmic alpha-fetoprotein functions as a co-repressor in RA-RAR signaling to promote the growth of human hepatoma Bel 7402 cells. Cancer Lett 285: 190-199, 2009.

41. Li M, Li H, Li C, Zhou S, Guo L, Liu H, Jiang W, Liu X, Li P, McNutt MA and Li G: Alpha fetoprotein is a novel protein-binding partner for caspase- 3 and blocks the apoptotic signaling pathway in human hepatoma cells. Int J Cancer 124: 2845-2854, 2009. 